\section{Multicusp ion source with external RF antenna for production of protons.}

S. K. Hahto*, S. T. Hahto, Q. Ji, K. N. Leung and S. Wilde, Lawrence Berkeley National Laboratory, 1 Cyclotron Road, Berkeley, CA 94720 and

E. L. Foley, F. M. Levinton and L. R. Grisham, Plasma Physics Laboratory, Princeton University, Princeton, NJ 08543

\section{Abstract}

Proton beams are needed in neutral beam injection for fusion research conducted at Princeton Plasma Physics Laboratory (PPPL). High proton fraction, low axial energy spread, current density in excess of $30 \mathrm{~mA} / \mathrm{cm}^{2}$ and a parallel ion beam are the requirements for the ion source/extraction system. A multicusptype ion source with an external RF antenna was constructed at Lawrence Berkeley National Laboratory (LBNL). Proton fraction of $85 \%$ and proton current density of $32 \mathrm{~mA} / \mathrm{cm}^{2}$ was achieved at $1.8 \mathrm{~kW}$ of RF power. Plasma parameters were measured with an RF compensated Langmuir probe.

\section{INTRODUCTION}

Multicusp-type ion sources are widely used to form positive ions. The source offers large, uniform plasma with a magnetic field free region in the middle of the source. Traditionally the plasma is created either by filament discharge ${ }^{1}$ or by an RF antenna placed inside the ion source chamber ${ }^{2}$. For the proton beams used in neutral beam injection to fusion reactor research conducted at PPPL the extracted proton beam is analyzed by the means of motional Stark effect with laserinduced fluorescence (MSE-LIF). The diagnostic is expected to enable the measurement of magnetic field magnitude and pitch angle in hot plasmas with fields as low as 0.1 T. In MSE-LIF it is essential that the ion beam axial energy spread is as low as possible and that the proton fraction of the extracted hydrogen ion beam is maximized. The ion beam should also be parallel when entering the neutralizer cell. Extracted current densities should be as high as possible at a beam energy of $35 \mathrm{keV}$ used in the diagnostic beam line at PPPL. A multicusp-type ion source with an external RF antenna and a magnetic filter was constructed and tested at LBNL before delivering it to PPPL. The compact source design means that RF powers below $2 \mathrm{~kW}$ produce intense enough ion beam, and the external antenna geometry removes all wearing parts from inside the plasma chamber and thus should give a long service interval and lifetime for the ion source.

\section{ION SOURCE}

\section{A. Preliminary tests}

A test source was constructed for testing of the external antenna operation. A quartz tube of $20 \mathrm{~mm}$ in length and $75 \mathrm{~mm}$ in diameter was used as a plasma chamber. A copper tube was wrapped around the quartz to form the RF antenna. Figure 1 shows a schematic of the test source.

*email: skhahto@lbl.gov

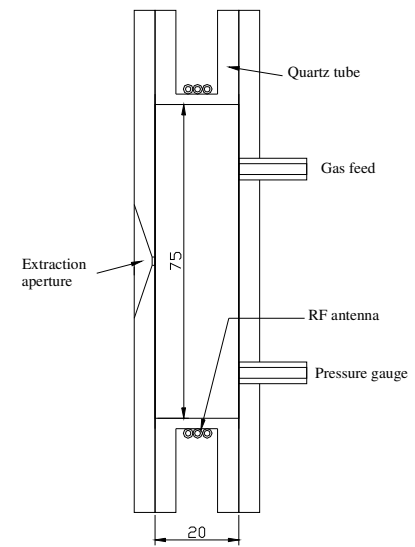

Figure 1. A schematic of the external antenna test source.

The test source was operated with $13.56 \mathrm{MHz} \mathrm{RF}$ frequency and up to $2 \mathrm{~kW}$ of RF power. The extracted hydrogen spectrum is shown in figure 2 . The proton fraction of the extracted hydrogen ions was $75 \%$. The proton fraction of the total current, including impurities such as $\mathrm{H}_{2} \mathrm{O}$, was $50 \%$.

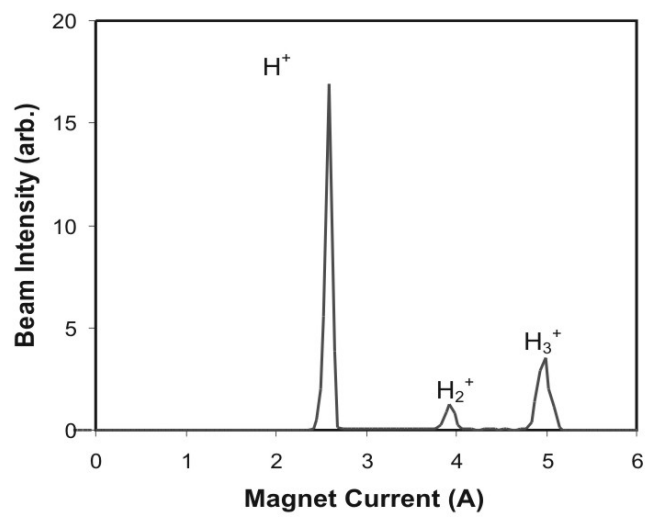

Figure 2. Extracted hydrogen ion spectrum at $1.8 \mathrm{~kW}$ RF power. Impurities are not shown.

Figure 3 shows the extracted total ion current density as a function of RF power.

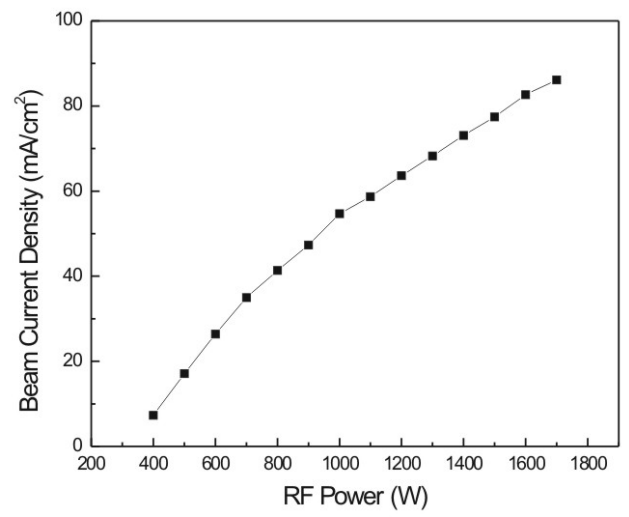

Figure 3. Extracted total ion current as a function of the RF power. 
From figure 3 it can be seen that a total current density of $80 \mathrm{~mA} / \mathrm{cm}^{2}$ was extracted from the test source at $1800 \mathrm{~W}$ of $\mathrm{RF}$ power. This corresponds to $40 \mathrm{~mA} / \mathrm{cm}^{2}$ proton current density. Based on the preliminary tests a multicusp- type ion source with an external RF antenna and a magnetic filter field inside the plasma chamber was designed. A target of $30 \mathrm{~mA} / \mathrm{cm}^{2}$ of proton current density and $80 \%$ proton fraction was set for the multicusp source.

\section{B. External RF antenna multicusp source}

A schematic of the constructed multicusp ion source is presented in figure 4 . The source chamber is $87 \mathrm{~mm}$ long and consists of 3 separate parts. A quartz cylinder with $60 \mathrm{~mm}$ inner diameter was sandwiched between two copper chambers with 75 mm inner diameter. The RF antenna was a 2.5 turn, water cooled copper tube wrapped around the quartz cylinder. The multicusp magnetic field confining the plasma was created with $14 \mathrm{SmCo}$ magnet rows that were installed into both copper chambers. 2 magnets were also installed to the back plate to complete the cusp lines in the back of the source and to prevent the flow of plasma to the laser input port extending from the back plate. In front of the extraction aperture two smaller SmCo magnet bars were installed to create a transverse filter magnetic field. The filter is needed in order have a uniform axial plasma potential distribution on the discharge region of the ion source, where most of the ionization takes place in the presence of the filter field. This minimizes the axial energy spread of the extracted ions and improves the proton fraction in the beam ${ }^{3,4}$. The 13.56 $\mathrm{MHz} \mathrm{RF}$ generator and the accompanying inductive matching network were operated at the ion source potential and the ion source was fully shielded to prevent the coupling of the RF into the extraction electrodes, which has been observed to increase the axial energy spread of the extracted ion beam ${ }^{5}$.

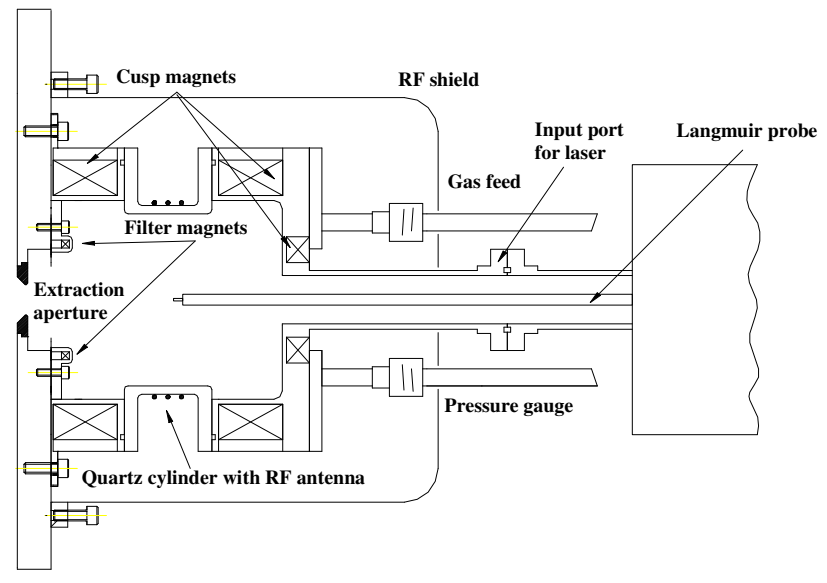

Figure 4: The $75 \mathrm{~mm}$ diameter, $87 \mathrm{~mm}$ long multicusp source with an external RF antenna

The measured transverse filter magnetic field along the ion source center axis is plotted in figure 5. It had a maximum value of 90 gauss and FWHM of $16 \mathrm{~mm}$.

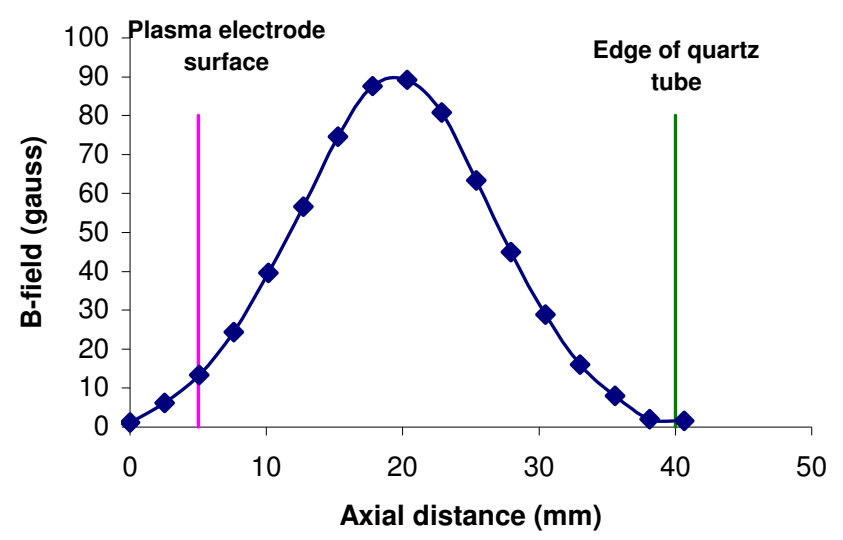

Figure 5: The filter magnetic field measured along the center axis of the ion source.

\section{EXTRACTION SIMULATIONS}

The extraction system was designed to provide a parallel beam with $35 \mathrm{~mA}$ proton current and $35 \mathrm{keV}$ beam energy at $19 \mathrm{~cm}$ downstream from the extraction aperture. An existing 3-electrode extraction system of the filament driven proton source at PPPL was utilized. PBGUNS ion optical simulation code ${ }^{6}$ was used in the simulations. Figure 6 show the PBGUNS simulation of the optimized extraction geometry with $99 \%$ beam space charge neutralization starting from the ground electrode.

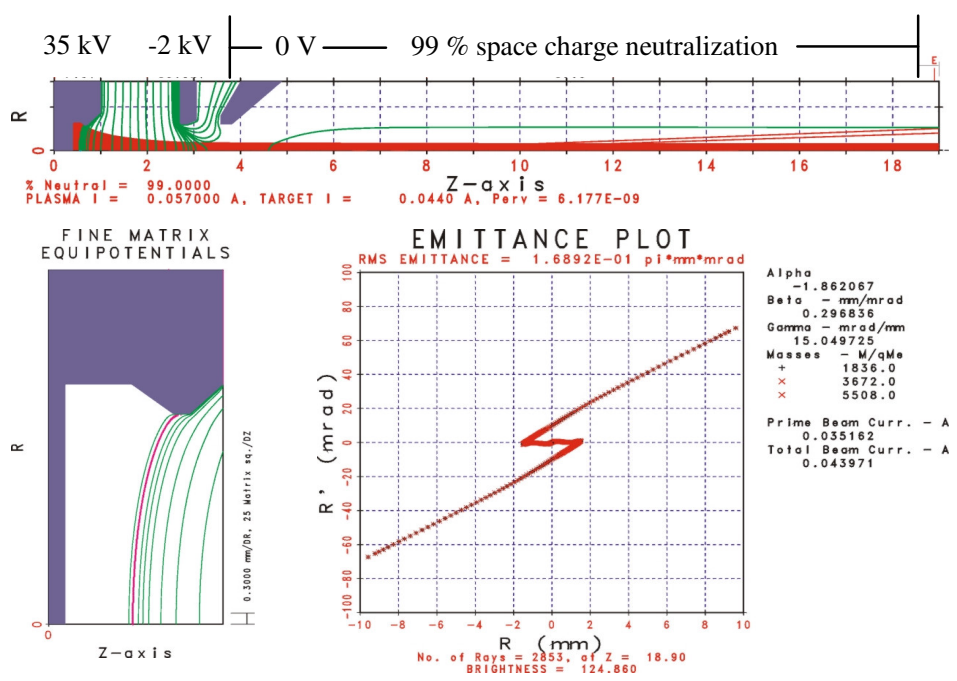

Figure 6: (Top) PBGUNS simulation of the $35 \mathrm{keV}, 44 \mathrm{~mA}$ positive hydrogen beam with $80 \%$ proton fraction $(35 \mathrm{~mA})$, (bottom) plasma meniscus and the beam emittance pattern at 19 $\mathrm{cm}$ downstream from the plasma electrode. Over $98 \%$ of the beam is contained in the parallel, $3.8 \mathrm{~mm}$ diameter beam core. The extraction aperture was $11.4 \mathrm{~mm}$ in diameter and the extraction gap was $17 \mathrm{~mm}$. 


\section{ION CURRENT AND SPECIES MEASUREMENTS}

The ion source performance was characterized by measuring the ion beam mass spectrum, total ion beam current and the axial plasma parameters. The ion source was installed in a test stand equipped with a $500 \mathrm{l} / \mathrm{s}$ turbomolecular pump. The total ion current was measured with a movable, secondaryelectron-suppressed Faraday cup. When ion beam mass spectrum was measured, the Faraday cup was retracted and the ion beam was focused into a magnetic-deflection mass spectrometer with an electrostatic einzel lens. The measurement setup used in the tests at LBNL was the same system that was used on a previous multicusp ion source experiment ${ }^{7}$. The beam was extracted from a $2 \mathrm{~mm}$ extraction aperture with an extraction gap of $4.5 \mathrm{~mm}$.

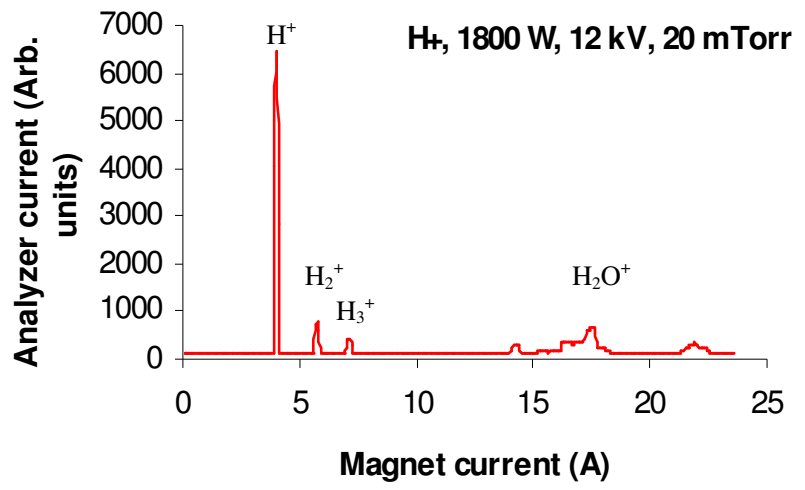

Figure 7: Measured hydrogen mass spectrum at $1800 \mathrm{~W}$ of RF power, $12 \mathrm{kV}$ extraction voltage and 20 mTorr source pressure

Figure 7 shows a typical hydrogen ion spectrum measured at $1800 \mathrm{~W}$ of RF power, $12 \mathrm{kV}$ of extraction voltage and 20 mTorr source pressure. About $85 \%$ of the positive hydrogen ions were $\mathrm{H}^{+}, 10 \% \mathrm{H}_{2}{ }^{+}$and $5 \% \mathrm{H}_{3}{ }^{+}$. Some impurities can be seen in the spectrum, mainly water. The proton fraction was more than 70 $\%$ if impurities were included in the species calculation. The level of impurities went down when the source was operated a few hours. The source will have a larger extraction aperture when it is installed at PPPL, which should speed up the cleanup of the source due to the greater gas flow through the plasma chamber.

Figure 8 shows the measured total ion current at two different RF powers as a function of the extraction voltage.

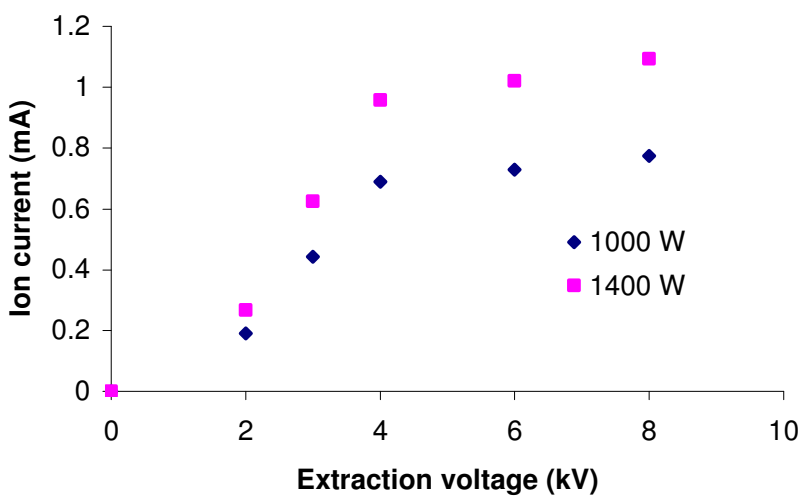

Figure 8: Measured total ion current as a function of the extraction voltage at $1000 \mathrm{~W}$ and $1400 \mathrm{~W}$ of RF power and 20 mTorr source pressure.
As can be seen, the ion current saturated at $\sim 4 \mathrm{kV}$ extraction voltage. Figure 9 shows the total ion and proton current densities as a function of RF power at 20 mTorr source pressure. The fraction of protons was determined from the mass spectrums for each RF power.

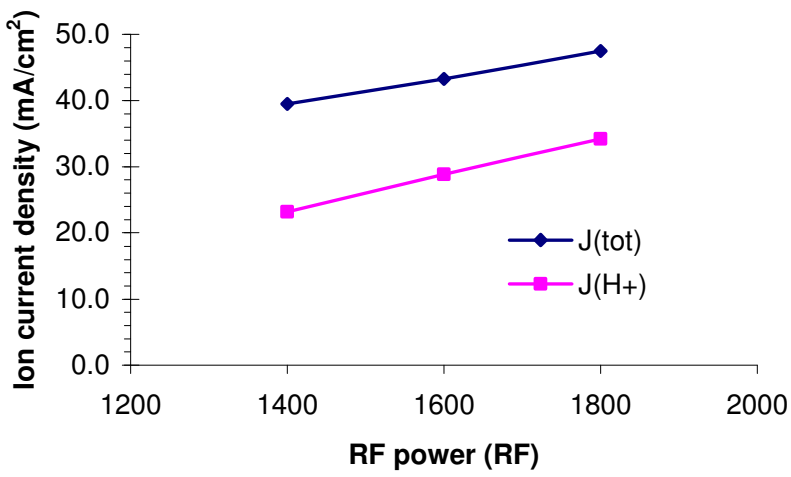

Figure 9: The measured proton and total ion current densities as a function of RF power at 20 mTorr source pressure.

From figure 9 it can be seen that a proton current density of 32 $\mathrm{mA} / \mathrm{cm}^{2}$ was achieved at $1800 \mathrm{~W}$ of RF power. This fulfills the proton current requirement set for the source.

Figure 10 shows the measured ion species fractions as a function of RF power. The proton fraction went up from $75 \%$ to $85 \%$ when the RF power increased from $1400 \mathrm{~W}$ to $1800 \mathrm{~W}$. This result met the goal set for the proton fraction.

The source operated optimally around 20 mTorr pressure. When the pressure is reduced, the matching conditions changed and the transformer turn ratio had to be adjusted. This prevented reliable comparison of source performance at different source pressures and the source operation was optimized for pressures around 20 mTorr.

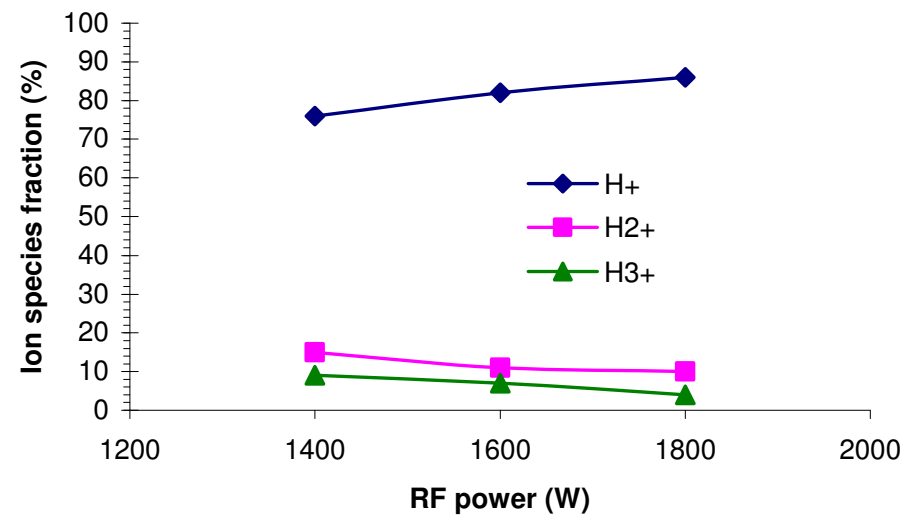

Figure 10: Hydrogen species fraction as a function of RF power at $20 \mathrm{mTorr}$ source pressure.

\section{PLASMA PROBE MEASUREMENTS}

A commercially available, RF compensated Langmuir probe system was used to make measurements of axial profiles of ion density, electron temperature, and plasma potential. A SmartProbe with Auto-Linear Drive mechanism from Scientific Systems ${ }^{8}$ was mounted on the laser inlet port of the source. 
The probe tip was a $0.2 \mathrm{~mm}$ radius tungsten wire of 1 $\mathrm{mm}$ length. The data was analyzed with the SmartProbe software, SmartSoft Version 3.31. The software determined the plasma potential by approximating the 'knee' of the probe characteristic as the intersection of lines fit to the regions of electron collection and electron saturation. As an estimate of the error in the analysis of the probe characteristics, the data was also fit for the best agreement in the ion saturation and electron collection regions of the probe characteristic only. The plasma potential was calculated both directly from the fits, and by using the floating potential and electron temperature, as $V_{p}=V_{f}$ $+3.3 \mathrm{~T}_{\mathrm{e}}$. The representative error bars in Figure 11 represent the largest discrepancy between the calculations, and those in Figure 12 represent the standard deviation of the values obtained from the various methods of calculating plasma potential. They do not account for systematic error, which is not known, or for variation in measured probe characteristics at the same plasma conditions, which was seen to be considerably smaller.

Probe scans were taken as plasma conditions varied with neutral gas pressure and RF power. Measured values and trends for the plasma parameters were consistent with those calculated from a cylindrical discharge model, where the plasma itself is considered to be contained within a cylindrical volume of uniform bulk plasma parameters, including density, electron temperature, and plasma potential.

The ion density $n_{i}$ increased with both RF power and neutral gas pressure, as would be expected. The maximum ion density measured was $2 \times 10^{12} \mathrm{~cm}^{-3}$, at $1400 \mathrm{~W}$ input power and $30 \mathrm{mT}$ source pressure, with values of $\mathrm{n}_{\mathrm{i}} \approx 10^{11} \mathrm{~cm}^{-3}$ more typical for operation at $1000 \mathrm{~W}$ input power and $12 \mathrm{mT}$. Both the plasma potential and the electron temperature dropped with increasing pressure, and stayed constant as the RF power varied, as expected. Figure 11 shows the axial profile of the measured ion density and electron temperature in the source.

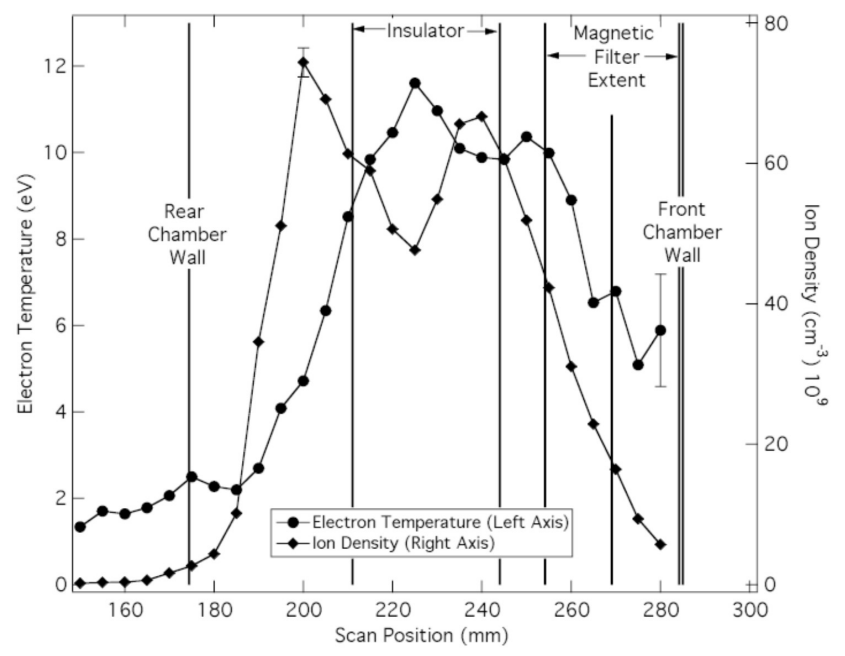

Figure 8: Axial variation of electron temperature and ion density in source with $1000 \mathrm{~W}$ RF power and 12 mTorr source pressure.

The motional Stark effect diagnostic for which the source will be used is very sensitive to the axial energy spread of the final neutral beam. Laser-induced fluorescence measurements will be performed on the beam, with the laser wavelength matched to the Doppler-shifted H $\alpha$ spectral line.
Any axial energy spread in the beam will result in broadening of the Doppler-shifted line, and is undesirable. Previous work has correlated axial energy spread with variation in plasma potential in the neutral beam source ${ }^{3}$, making the measurement of the variation of plasma potential in the source of particular interest. Figure 12 plots the measured axial plasma potential distribution in the source. The variation of plasma potential is seen to be on the order of $\sim 5 \mathrm{eV}$. This is comparable to the lowest energy spreads reported in rf sources, in the order of $3 \mathrm{eV}^{5}$. Factors such as RF coupling and accelerating power supply ripple may also contribute to the beam's axial energy spread.

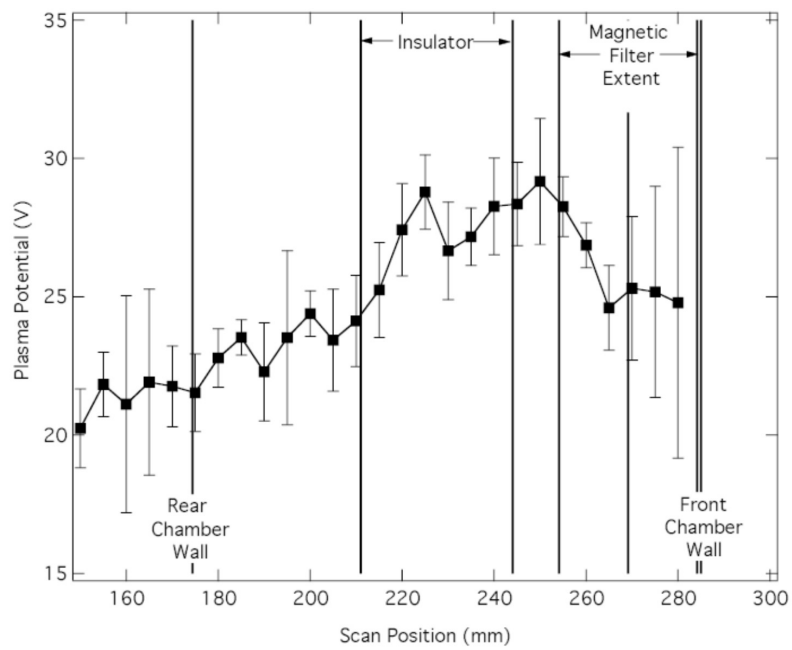

Figure 12: Axial variation of the plasma potential at $1000 \mathrm{~W} \mathrm{RF}$ power and 12 mTorr source pressure.

\section{ACKNOWLEDGEMENTS}

This work is supported in part by Nova Photonics, Inc. and by the U.S. Department of Energy under Contract No. DEAC03-76SF00098. One of the authors (S. K. Hahto) would like to thank the Finnish Academy of Science and Letters for additional support. E. L. Foley gratefully acknowledges the support of a Hertz Foundation Graduate Fellowship.

${ }^{1}$ K. W. Ehlers and K. N. Leung, Rev. Sci. Instrum. 54 (10), pp. 1296 (1983)

${ }^{2}$ D. Wutte , S. Freedman, R. Gough, Y. Lee, M. Leitner, K.N. Leung, C. Lyneis, D.S. Pickard, M.D. Williams, Z.Q. Xie, Nucl. Instr. and Meth. in Phys. Res. B 142, pp. 409 (1998)

${ }^{3}$ Y. Lee, R.A. Gough, W. B. Kunkel, K. N. Leung and L. T. Perkins, Nucl. Instr. and Meth. In Phys. Res. A 374,pp. 1 (1996)

${ }^{4}$ K. W. Ehlers and K. N. Leung, Rev. Sci. Instrum. 52(10), pp. $1452(1981)$

${ }^{5}$ Y. Lee, R. A. Gough, W. B. Kunkel, K. N. Leung, L. T. Perkins, D. S. Pickard, L. Sun, J. Vujic, M. D. Williams, and D. Wutte, Rev. Sci. Instrum. 68 (3), pp. 1398 (1997)

${ }^{6}$ J. E. Boers, Proc. of the 1995 Particle Accelerator Conference, pp. 2312 (1995) 
${ }^{7}$ S. K. Hahto, S. T. Hahto, J. W. Kwan, K. N. Leung and L. R. Grisham, Rev. Sci. Instrum. 74(6), pp. 2987 (2003)

${ }^{8}$ Scientific Systems Ltd., Unit 3, Howth Junction Business Park, Kilbarrack, Dublin 5, Ireland. SmartProbe Automatic Langmuir Probe User Manual, 2002.info@scisys.com, www.scisys.com. 